\title{
Comparative Study of the Effectiveness of Matrix Training and tDCS Treatments on Positive and Negative Affects and Craving in Substance Abusers
}

\author{
Salem Mosavi ${ }^{1}$, Parvin Ehtesham Zadeh $^{2 *} \mathbb{D}$, Zahra Eftekhar Saadi² ${ }^{(\mathbb{D}}$, Alireza Heydarei ${ }^{(\mathbb{D}}$ \\ 1 Department of Psychology, Khorramshahr Branch, Islamic Azad University, Khorramshahr, Iran \\ 2 Department of Psychology, Ahvaz Branch, Islamic Azad University, Ahvaz, Iran
}

\author{
*Corresponding author: \\ Parvin Ehtesham Zadeh, Department \\ of Psychology, Ahvaz Branch, Islamic \\ Azad University, Ahvaz, Iran \\ Tel: 09166036112 \\ Email:ehtesham@iauahvaz.ac.i
}

Received: 09 Jan. 2020

Accepted: 10 May. 2020

ePublished: 01 Aug. 2020

\begin{abstract}
Background and Objective: Substance dependence is acknowledged as one of the major social and health issues inflicting severe and profound physical and psychological harm, as well as numerous social damages, such as divorce and unemployment. The present study aimed to make a comparison between the effectiveness of Matrix training and transcranial direct current stimulation (tDCS) treatments on positive and negative affects and craving in substance abusers who referred to Ahwaz addiction treatment centers within 2018-2019.

Materials and Methods: The present semi-experimental study was conducted using a pre-test posttest control group design with a two-month follow-up. The study population consisted of all substance abusers who referred to Ahwaz addiction treatment centers within 2018-2019. A total of 60 volunteers were selected by voluntary sampling method and randomly assigned to Matrix training $(n=20)$, tDCS $(n=20)$, and control $(n=20)$ groups. Data were collected by The Positive and Negative Affect Schedule (PANAS) and Desire For Drug Questionnaire and were analyzed in SPSS software (version 22).

Results: Based on the obtained results, Matrix, tDCS, and control groups were significantly different in terms of positive and negative affects and craving $(\mathrm{P}<0.001)$. Moreover, it was found that Matrix training and tDCS were effective on positive and negative affects and cravings $(P<0.001)$; nonetheless, no significant difference was observed between the matrix and tDCS groups $(P>0.05)$. Conclusion: Generally speaking, it can be concluded that Matrix training and tDCS methods are equally effective in emotions and craving.

Keywords: Affect, Craving, Drug users, Transcranial direct current stimulation
\end{abstract}

\section{Background}

Substance dependence, also known as drug dependence is recognized as one of the major health and social issues which pose a palpable risk to different communities in the present century. It is a matter of great intellectual concerns and one of the most unfortunate social harms. This disorder can inflict severe and profound physical and psychological harm, as well as numerous social damages, such as divorce and unemployment [1]. According to global statistics released within 20062015, the number of problem drug users worldwide has increased by $23 \%$. It was estimated that the global prevalence rate of drug abuse in 2006 was about $9.4 \%$, and it reached $39.5 \%$ in 2015 [2]. Several factors are involved in the onset and persistence of addiction, the most important of which are mental disorders and emotional problems [3].

As documented in various studies, negative emotions and drug-related disorders go hand-in- hand with each other, and those who are emotionally challenged are more susceptible to drug and alcohol dependence[4]. An increase in drug use provokes negative emotions, which in turn, fuel drug addiction [5] since negative emotions have been acknowledged as a key factor in increasing drug use [6]. The application of effective emotional adjustment skills in such situations can improve one's emotions and helps them to cope [7]. The main incentive for drug use is the utilization of their psychological properties to regulate and modify negative emotions and achieve emotional stability [8].

Craving is recognized as another crucial factor responsible for the onset and persistence of addiction and addictive behaviors in drugdependent individuals [9]. This feeling involves an extensive range of phenomena, such as expectancies about the reinforcing effects and a strong tendency for substances. Craving is a powerful feeling and an 
urgent desire for something that makes it impossible to focus on anything other than the subject in question. [10]. In various studies, this strong urge was found to be the main incentive for continued drug abuse and addiction relapse. Based on the findings of multiple studies, it can be argued that automatic or non-automatic cognitiveemotional processes control craving. Craving theories generally emphasize that cravings in individuals are related to the activation of emotions and motivations for substances seeking[11].

The numerous psychological and physical effects and consequences of drug abuse highlight the need for various medical and psychological interventions. Since addicts respond differently to therapies, appropriate therapies should be considered for each individual. Matrix, transcranial direct-current stimulation (tDCS), and Matrix and tDCS in combinations are among the widely used therapies . The Matrix Model is a structured treatment model that provides clients with useful information on how to lead a healthy life [12]. The results of previously conducted studies show that Matrix treatment is effective in the improvement of stress coping strategies in addicts [13] . One of the major challenges patients face during treatment is addiction relapse, and some addicts return to their initial routine use of substance after the treatment. Multiple studies have investigated patients' selfregulation [14] ,clients' unstable quality of life [15], and the use of the synchronous change pattern with the Matrix model.

The transcranial direct current stimulation (tDCS) method is one of the recent therapies used for the treatment of addiction. It is a non-invasive, painless, and safe brain stimulation method which is used to modulate cortical excitability and psychiatric disorders [16]. Although the precise mechanism of tDCS has not yet been elucidated, it is thought to be able to differentially affect spontaneous cortical excitability and spontaneous neuronal firing which makes this technique an attractive tool for the treatment of psychiatric illnesses. Some studies have pointed to the positive effects of this method on the treatment of depression, Parkinson's disease, and addiction (especially alcoholism). Based on the findings of recently conducted studies, tDCS reduces cravings for food and alcohol [17].

\section{Objectives}

The present study aimed to compare the effectiveness of Matrix and tDCS treatments on positive and negative emotions and craving in substance abusers.

\section{Materials and Methods}

The present semi-experimental study was conducted using a pre-test post-test control group design with a two-month follow-up. The study population consisted of all substance abusers who referred to Ahwaz addiction treatment centers within 2018-2019. The inclusion criteria were as follows: 1)drug addiction, 2) literacy, 3)no psychiatric disorders, 4) impulse control, 5) nonuse of antipsychotics, 6) having a family (not being homeless), and 7) willingness to participate in the study. A total of 60 volunteers were selected by voluntary sampling method and randomly assigned to Matrix training $(\mathrm{n}=0)$, tDCS $(\mathrm{n}=20)$ and control $(n=20)$ group. The subjects in the tDCS group were treated with tDCS for 10 sessions. To perform this procedure, the anode (excitation) electrode was positioned in the posterior region of the left forebrain cortex (F3), and the cathode electrode (inhibitor) was placed on the posterior region of the right forebrain cortex (F4), and 2 Milli Amperof direct current was passed through the skull for $20 \mathrm{~min}$.

The Positive and Negative Affect Schedule or (PANAS)

This scale is a 20 -item measurement tool designed to measure negative and positive affects [18].In this questionnaire, each subscale consists of 10 items which are scored on a five-point Likert scale $(1=$ very low to $5=$ very high $)$. This questionnaire is desirable in terms of reliability and validity. The internal consistency coefficients of this scale were calculated as 0.87 and 0.88 for negative and positive Panas, respectively. Moreover, the test-retest reliability was obtained at 0.71 for negative Panas and 0.68 for positive Panas .The validity of this questionnaire was also estimated appropriately by the calculation of the correlation between negative and positive Panas with some of the research tools that measure the structures associated with these two scales, such as anxiety.For instance, the results showed that the negative Panas correlation with the Hopkins symptom checklist was calculated at 0.72 , and the positive Panas correlation with the manifest anxiety scale was measured at -0.35 [18]. In studies conducted by Kaviani, Soleimani, Sajjadi, and Nazari [19], the validity coefficient was reported as 0.77 for positive Panas and 0.83 for negative Panas.

Desire For Drug Questionnaire

This questionnaire which was first designed by Franken et al. in 2002 is used for substance craving measurement [20]. This 14-item questionnaire is comprised of three different sub-scales; "desire and 
intention," "negative reinforcement," and "loss of control .This questionnaire is rated on a 6-point Likert scale ranging from $-3=$ complete agreement to $+3=$ complete dissent. A high and positive score in this questionnaire signifies more craving. In a study conducted by Alizadeh on opioid abusers, including crack and heroin, [21], using the internal consistency method, Cronbach's alpha coefficients for the three subscales of this questionnaire were reported as $0.84,0.79$, and 0.89 respectively, and it was obtained at 0.86 for the total scale. In addition, for methamphetamine abusers, coefficient values for the three mentioned subscales were calculated at $0.78,0.79$, and 0.89 , respectively.

The obtained data were analyzed in SPSS software (version 24) using descriptive statistics (e.g., frequency, percentage, mean, standard deviation) and inferential statistics (e.g., multivariate and univariate analysis of covariance), post hoc test, and their assumptions .The significance level was considered to be $\alpha=0.05$.

\section{Results}

The mean age scores of participants were reported as 38.58 (7.69), 36.44 (7.79), and 37.5 (7.84) in the Matrix, tDCS, and control groups, respectively. The subjects were the age range of 25-40 years. Mean and standard deviation of research variables are presented in Table 1.

According to the Kolmogorov-Smirnov test, the significant level in the case and control groups showed the normal distribution of the scores in the study population. According to the results of the present study, the relationship of craving with positive and negative affects at post-tests was significant $(\mathrm{P}<0.05)$, and the assumption of linearity was observed. Moreover, since the correlation coefficients between the variables are less than 0.9, it can be argued that the assumption of multiple nonlinearities between the covariates is considered. The results of the variance homogeneity test showed that due to the non-significance of the Levin test, the covariance analysis test could be used. The box test was utilized to assess the assumption of homogeneity of covariances in the present study. The results of this test revealed that there was no significant homogeneity of covariances assumption $\left(\mathrm{P}=0.652, \mathrm{~F}=0.854, \mathrm{Box}^{\prime} \mathrm{s}=10.324\right)$; therefore, the assumption difference between covariances is confirmed. Furthermore, according to the results, the interaction of regression slopes with positive and negative affects and craving within the groups was not significant, followed by the confirmed assumption of regression slope homogeneity.

According to the results presented in Table 2, it was found that all four multivariate statistics, namely Pillai's Trace, Wilks Lambda, Hotelling's Trace, and Roy's Largest Root, were significant. Based on the results, in the post-test stage, there was a significant difference between the subjects who received the intervention (case group) and the subjects who did not receive the intervention (control group) at least in one of the dependent variables $(\mathrm{P}<0.01)$. An effect or difference of 0.52 indicates that $52 \%$ of the individual differences in post-test scores were associated with the effect of Matrix and tDCS treatments.

As illustrated in Table 3, the $\mathrm{F}$ ratio of univariate analysis of covariance for positive affect $(\mathrm{F}=23.81$ and $\mathrm{P}=0.001)$, negative affect $(\mathrm{F}=19.92$ and $\mathrm{P}=0.001)$, and craving $(\mathrm{F}=41.96$ and $\mathrm{P}=0.001)$. The results presented in Table 4 show that the Matrix, tDCS, and control groups were significantly

Table 1. Mean and standard deviation of positive and negative affects, as well as craving in the matrix, tDSC, and control groups in the pretest and post-test stages

\begin{tabular}{|c|c|c|c|c|c|c|}
\hline \multirow{2}{*}{ Variables } & \multirow{2}{*}{ Groups } & \multicolumn{2}{|c|}{ pre-test } & \multicolumn{2}{|c|}{ post-test } & \multirow{2}{*}{ p-value } \\
\hline & & Mean & SD & Mean & SD & \\
\hline \multirow{3}{*}{ Positive Affect } & Matrix group & 20.65 & 3.77 & 34.05 & 8.44 & 0.001 \\
\hline & TDCS group & 17.95 & 3.13 & 29.90 & 6.13 & 0.001 \\
\hline & Control group & 21.85 & 2.85 & 20.23 & 4.42 & 0.598 \\
\hline \multirow{3}{*}{ Negative Affect } & Matrix group & 39.00 & 3.16 & 26.00 & 7.02 & 0.001 \\
\hline & TDCS group & 39.15 & 3.64 & 26.70 & 7.80 & 0.001 \\
\hline & Control group & 38.50 & 2.03 & 37.65 & 5.31 & 0.714 \\
\hline \multirow{3}{*}{ Craving } & Matrix group & 65.00 & 3.50 & 47.10 & 8.74 & 0.001 \\
\hline & TDCS group & 64.20 & 3.34 & 45.65 & 9.44 & 0.001 \\
\hline & Control group & 63.85 & 3.06 & 66.10 & 6.60 & 0.134 \\
\hline
\end{tabular}

Table 2. Results of multivariate analysis of covariance on post-test scores of positive and negative affects, and craving in case and control groups

\begin{tabular}{|c|c|c|c|c|c|c|c|}
\hline Test & Value & $F$ & df Hypothesis & df Error & p-value & Effect size & Power \\
\hline Pillai's Trace & 1.42 & 6.86 & 18 & 201 & 0.001 & 0.38 & 1.00 \\
\hline Wilks Lambda & 0.109 & 12.20 & 18 & 184 & 0.001 & 0.52 & 1.00 \\
\hline Hotelling's Trace & 6.012 & 21.26 & 18 & 191 & 0.001 & 0.66 & 1.00 \\
\hline Roy's Largest Root & 5.648 & 63.07 & 6 & 67 & 0.001 & 0.85 & 1.00 \\
\hline
\end{tabular}


Table 3. Results of ANCOVA on post-test scores of positive and negative affects and craving

\begin{tabular}{lccccccc}
\hline Source & Variables & Sum of Squares & df & Mean Squares & F & p-value & Effect size \\
\hline \multirow{3}{*}{ Groups } & Positive Affect & 2222.72 & 3 & 740.90 & 23.81 & 0.001 & 0.50 \\
& Negative Affect & 2344.22 & 3 & 781.40 & 19.92 & 0.001 & 0.46 \\
& Craving & 6607.91 & 3 & 2202.63 & 41.96 & 0.001 & 0.64 \\
\hline
\end{tabular}

Table 4. Results of the Bonferroni post hoc test to compare the difference between three groups in the post-test

\begin{tabular}{llccc}
\hline Variables & Comparison groups & Mean difference & Standard error & p-value \\
\hline \multirow{3}{*}{ Positive Affect } & Group 1-Control group & 11.02 & 1.88 & 0.001 \\
& Group 2-Control group & 7.69 & 2.14 & 0.001 \\
& Group 1- Group 2 & 3.33 & 1.99 & 0.100 \\
Negative Affect & Group 1-Control group & 11.40 & 2.11 & 0.001 \\
& Group 2-Control group & 10.55 & 2.41 & 0.001 \\
Craving & Group 1- Group 2 & 0.846 & 4.24 & 0.707 \\
& Group 1-Control group & 19.40 & 2.44 & 0.001 \\
& Group 2-Control group & 18.75 & 2.78 & 0.001 \\
\hline
\end{tabular}

different in terms of dependent variables (e.g., positive and negative affects and craving). In the remainder of this study, the results of the Bonferroni post hoc test were compared to mean differences, standard error, and significance levels of positive and negative affects and craving in the Matrix, tDCS, and control groups.

The results displayed in Table 5 indicate that Matrix, tDCS, and control groups were significantly different regarding positive and negative affects and craving $(\mathrm{P}<0.001)$. As evidenced by the results of the present study, Matrix training and tDCS were effective on positive and negative affects and cravings $(\mathrm{P}<0.001)$; nonetheless, no significant difference was observed between the matrix and tDCS groups $(\mathrm{P}>0.05)$.

\section{Discussion}

The findings of the present study pointed to a significant difference between the case and control groups in positive and negative affects and craving. Moreover, the results of the Bonferroni table demonstrated a significant difference between the tDCS and control groups in the post-test of craving. Considering the mean craving scores presented in the descriptive table, it can be concluded that direct transcutaneous electrical stimulation of the brain is effective on positive and negative affects and craving in substance abusers. These results are in line with those reported by Boggio et al. [22], Fecteau et al. [23], and Batista et al. [24].

It can be explained that although the mechanism of action of tDCS is not well-documented, the available evidence links the potential changes induced by transcranial magnetic stimulation (TMS) to the effects on neurotransmitters and neuroplasticity of nerve cells. [25, 26]. Owing to its effect on cortical excitability and dopaminergic transducers, TMS has been introduced as a tool for the treatment of addiction disorder in the current study. Previous studies have shown that repeated high-frequency ultrasound magnetic stimulation alters dopamine transducers and amplifies them in subcutaneous structures [27]. Moreover, in order to explain these results, we can refer to the reinforcement sensitivity theory, which is known as the neural adaptation pattern, and the consumption cravings are due to the involvement of the neural circuit, neural substrate, and brain reward systems. Long-term changes in cortical irritability due to repeated magnetic stimulation of transcranial neurotransmitter dopamine can be an explanation for the results, and the basic mechanisms of cravings are due to the high sensitivity of dopamine neurotransmitter, which leads to increased drug excitability [28].

Previously conducted animal studies have demonstrated that anodic stimulation increases neuronal firing, and cathodic stimulation leads to opposite results. Therefore, it is assumed that either an increase in the right frontal or left frontal activity leads to a decrease in craving [29]. The posterior dorsal prefrontal region is one of the most important areas of the prefrontal cortex. It is responsible for identifying and defining actions, assessing future consequences of current behavior, and predicting social consequences [30]. As mentioned earlier, one possible mechanism that can stimulate this area to reduce craving is the enhancement of social control or participants' ability to suppress their desires. In addition, according to the results of previous studies, it can be stated that increased or decreased stimulation of the left or right frontal area can disrupt the balance of activity in the two hemispheres. Therefore, the stimulation of the dorsal parts of the left forehead and right frontal cortex can reduce the craving states.

The results of the present study indicated that the performed interventions were effective in the reduction of substance use and relapse in opiate 
dependents. This result is consistent with the findings reported by Ray et al. [31] and Chen et al. [32]. The goals pursued in the Matrix model include: 1) cutting down or stopping drug use, 2) absence of addiction relapse, 3) learning critical issues in addiction and re-entry, 4) providing guidance and support to clients, 5) training family members affected by addiction, 6) familiarity with self-help programs, and 7) monitoring by urine drug tests.

The results of the present study on the effect of Matrix Therapy on positive and negative affects showed that this method is effective in the reduction of negative affects and the enhancement of positive affects in addicts. In explaining this finding, it can be said that given that Matrix treatment respects autonomy and choice, changing health behaviors is facilitated [32]. One of the limitations of the present study is the limited number of addicted people under the study. It is recommended that studies with more samples be conducted to generalize these findings.

\section{Conclusions}

It can be concluded that Matrix training and tDCS methods are equally effective in emotions and craving.

\section{Compliance with ethical guidelines}

All ethical principles were considered in this research. The participants were informed about the purpose of the research and its stages. Informed consent was obtained from the subjects. They were also assured of the confidentiality of their information. Moreover, the subjects were free to withdraw from the study if desired. They were also informed that they would be provided with the results of the research. This study was extracted from a doctoral dissertation of counseling in Khorramshahr Branch, Islamic Azad University, Khorramshahr, Iran with the code of ethics(IR.IAU.AHVAZ.REC.1397.009) from the Ethics Committee of Islamic Azad University of Ahvaz.

\section{Acknowledgments}

The deepest appreciation goes to the participants who made a great contribution to this research project.

\section{Authors' contributions}

Conceptualization [Salem Mosavi], methodology [Parvin Ehtesham Zadeh], investigation [Zahra Eftekhar Saadi], writing the original draft [Alireza Heydarei], writing-review and editing, author names [all author], funding [all authors], resources [all author], Supervision [Parvin Ehtesham Zadeh].

\section{Funding/Support}

This research did not receive any specific grant from funding agencies in the public, commercial, or not-for-profit sectors.

\section{Conflicts of Interest}

The authors declare that they have no conflict of interest regarding the publication of the current study.

\section{References}

1. Berking $M$, Margraf $M$, Ebert $D$, Wupperman $P$, Hofmann SG, Junghanns K. Deficits in emotion-regulation skills predict alcohol use during and after cognitive-behavioral therapy for alcohol dependence. Journal of Consulting and Clinical Psychology. 2011; 79(3):307-18. [DOI:10.1037/ a0023421] [PMID] [PMCID]

2. Camprodon JA, Martínez-Raga J, Alonso-Alonso M, Shih MC, Pascual-Leone A. One session of high frequency repetitive transcranial magnetic stimulation (rTMS) to the right prefrontal cortex transiently reduces cocaine craving. Drug and Alcohol Dependence. 2007; 86(1):91-4. [DOI:10.1016/j.drugalcdep.2006.06.002] [PMID]

3. Feil J, Sheppard D, Fitzgerald PB, Yücel M, Lubman DI, Bradshaw JL. Addiction, compulsive drug seeking, and the role of frontostriatal mechanisms in regulating inhibitory control. Neuroscience \& Biobehavioral Reviews. 2010; 35(2):248-75. [DOI:10.1016/j.neubiorev.2010.03.001] [PMID]

4. Fischer B, Blanken P, Da Silveira D, Gallassi A, Goldner EM, Rehm J, et al. Effectiveness of secondary prevention and treatment interventions for crack-cocaine abuse: a comprehensive narrative overview of English-language studies. International Journal of Drug Policy. 2015; 26(4):35263. [DOI:10.1016/j.drugpo.2015.01.002] [PMID]

5. Hormes JM, Rozin P. Does "craving" carve nature at the joints? Absence of a synonym for craving in many languages. Addictive Behaviors. 2010; 35(5):459-63. [DOI:10.1016/j.addbeh.2009.12.031] [PMID]

6. Kassel JD, Evatt DP, Greenstein JE, Wardle MC, Yates MC, Veilleux JC. The acute effects of nicotine on positive and negative affect in adolescent smokers. Journal of Abnormal Psychology. 2007; 116(3):543-53. [DOI:10.1037/0021843X.116.3.543] [PMID]

7. Massah O, Effatpanah MO, Shishehgar SA. Matrix model for methamphetamine dependence among Iranian female methadone patients: the first report from the most populated Persian Gulf country. Iranian Rehabilitation Journal. 2017; 15(3):193-8. [DOI:10.29252/nrip.irj.15.3.193]

8. Measelle JR, Stice E, Springer DW. A prospective test of the negative affect model of substance abuse: moderating effects of social support. Psychology of Addictive Behaviors. 2006; 20(3):225-33. [DOI:10.1037/0893-164X.20.3.225] [PMID]

9. Michaud K, Grabherr S, Shiferaw K, Doenz F, Augsburger $M$, Mangin P. Acute coronary syndrome after levamisoleadultered cocaine abuse. Journal of Forensic and Legal Medicine. 2014; 21:48-52. [DOI:10.1016/j.jflm.2013.10.015] [PMID]

10. Rosselli M, Simmers CP. Effects of alcohol in chronic cocaine abuse: a follow up study. Journal of Drug Abuse. 2016; 2(1):1-11.

11. Suh JJ, Ruffins S, Robins CE, Albanese MJ, Khantzian EJ. Selfmedication hypothesis: Connecting affective experience and drug choice. Psychoanalytic Psychology. 2008; 25(3):518. [DOI:10.1037/ 0736-9735.25.3.518]

12. Tiffany ST, Drobes DJ. The development and initial validation of a questionnaire on smoking urges. British Journal of Addiction. 1991; 86(11):1467-76. [DOI:10.1111/ j.1360-0443.1991.tb01732.x] [PMID]

13. Watson D, Clark LA, Carey G. Positive and negative affectivity and their relation to anxiety and depressive disorders. Journal of Abnormal Psychology. 1988; 97(3): 346-53. [DOI:10.1037//0021-843x.97.3.346] [PMID]

14. Wing VC, Barr MS, Wass CE, Lipsman N, Lozano AM, Daskalakis ZJ, et al. Brain stimulation methods to treat tobacco addiction. Brain Stimulation. 2013; 6(3):221-30. [DOI:10.1016/j.brs.2012.06.008] [PMID]

15. Wupperman P, Marlatt GA, Cunningham A, Bowen S, Berking M, Mulvihill-Rivera $N$, et al. Mindfulness and modification therapy for behavioral dysregulation: Results from a pilot study targeting alcohol use and aggression in women. Journal of Clinical Psychology. 2012; 68(1):50-66. [DOI:10.1002/jclp.20830] [PMID]

16. Jones S, Lynam DR, Piquero AR. Substance use, personality, and inhibitors: Testing Hirschi's predictions about the reconceptualization of self-control. Crime \& Delinquency. 2015; 61(4):538-58. [DOI:10.1177/0011128711420109] 
17. Skjærvø I, Skurtveit S, Clausen T, Bukten A. Substance use pattern, self-control and social network are associated with crime in a substance-using population. Drug and Alcohol Review. 2017; 36(2):245-52. [DOI:10.1111/dar.12406] [PMID]

18. Walters KJ, Simons JS, Simons RM. Self-control demands and alcohol-related problems: Within-and between-person associations. Psychology of Addictive Behaviors. 2018; 32(6):573-82. [DOI:10.1037/adb0000387] [PMID] [PMCID]

19. Oliva A, Antolín-Suárez L, Rodríguez-Meirinhos A. Uncovering the link between self-control, age, and psychological maladjustment among Spanish adolescents and young adults. Psychosocial Intervention. 2019; 28(1):49-55. [DOI:10.5093/pi2019a1]

20. Hajihasani M, Shafiabadi A, Pirsaghi F, Kiyanipour O. Relationship between aggression, assertiveness, depression and addiction potential in female students of Allameh Tabbatabai. Knowledge \& Research in Applied Psychology. 2012; 13(49):65-74.

21. Yang C, Zhou Y, Cao Q, Xia M, An J. The relationship between self-control and self-efficacy among patients with substance use disorders: resilience and self-esteem as mediators. Frontiers in Psychiatry. 2019; 10:388. [DOI:10.3389/fpsyt.2019.00388] [PMID] [PMCID]

22. Boggio PS, Sultani N, Fecteau S, Merabet L, Mecca T, Pascual-Leone A, et al. Prefrontal cortex modulation using transcranial DC stimulation reduces alcohol craving: a double-blind, sham-controlled study. Drug and Alcohol Dependence. 2008; 92(1-3):55-60. [DOI:10.1016/j.drugal cdep.2007.06.011] [PMID]

23. Batista EK, Klauss J, Fregni F, Nitsche MA, NakamuraPalacios EM. A randomized placebo-controlled trial of targeted prefrontal cortex modulation with bilateral tDCS in patients with crack-cocaine dependence. International Journal of Neuropsycho-pharmacology. 2015; 18(12): pyv066. [DOI:10.1093/iinp/pyv066] [PMID] [PMCID]

24. Fecteau S, Agosta S, Hone-Blanchet A, Fregni F, Boggio P, Ciraulo D, et al. Modulation of smoking and decisionmaking behaviors with transcranial direct current stimulation in tobacco smokers: a preliminary study. Drug and Alcohol Dependence. 2014; 140:78-84. [DOI:10.1016/j. drugalcdep.2014.03.036] [PMID] [PMCID]

25. Karatay G, Baş NG. Factors affecting substance use and selfefficacy status of students in eastern Turkey. Ciencia \& Saude Coletiva. 2019; 24(4):1317-26. [DOI:10.1590/141381232018244.30252017] [PMID]

26. Elsner B, Kugler J, Mehrholz J. Transcranial direct current stimulation (tDCS) for upper limb rehabilitation after stroke: future directions. Journal of Neuroengineering and Rehabilitation. 2018; 15(1):106. [DOI:10.1186/s12984-0180459-7] [PMID] [PMCID]

27. Chaturvedi R, Kulandaivelan S, Malik M, Joshi S. Effect of transcranial direct current stimulation (TDCS) on pain in fibromyalgia-systematic review based on Prisma guidelines. Pain. 2018; 10(11):12.

28. Carter MJ, Smith V, Carlsen AN, Ste-Marie DM. Anodal transcranial direct current stimulation over the primary motor cortex does not enhance the learning benefits of selfcontrolled feedback schedules. Psychological Research. 2018; 82(3):496-506. [DOI:10.1007/s00426-017-0846-x] [PMID]

29. Khosravian B, Soleimani E. The effect of transcranial direct current stimulation (tDCS) on drug use craving and cognitive self-control of substance abusers. Research on Addition. 2018; 12(47):93-110.

30. Besharat MR, Karimi RM, Farahani $H$. The effectiveness of an augmented tDCS and ABMT on reducing food craving in obese individuals. Applied Psychological Research Quarterly. 2015; 6(3):19-37.

31. Ray MK, Sylvester MD, Helton A, Pittman BR, Wagstaff LE, McRae TR 3rd, et al. The effect of expectation on transcranial direct current stimulation (tDCS) to suppress food craving and eating in individuals with overweight and obesity. Appetite. 2019; 136:1-7. [DOI:10.1016/j.appet. 2018.12.044] [PMID] [PMCID]

32. Chen S, Jackson T, Dong D, Zhang X, Chen H. Exploring effects of single-session anodal tDCS over the inferior frontal gyrus on responses to food cues and food cravings among highly disinhibited restrained eaters: a preliminary study. Neuroscience Letters. 2019; 706:211-6. [DOI:10.1016/j. neulet.2019.05.035] [PMID] 